\title{
Does Endovascular Therapy Benefit Patients with Acute Ischemic Stroke?
}

Benton R. Hunter, MD (EBEM Commentator),

Shradha V. Shardhul, MBBS, DEM (EBEM Commentator)

\section{TAKE HOME MESSAGE}

In management of acute ischemic stroke, there is no evidence that endovascular therapy improves functional outcomes over that achieved with standard care.

\section{METHODS}

\section{Data Sources}

The authors searched MEDLINE, EMBASE, Cochrane Central Register of Controlled Trials, Cochrane Database of Systematic Reviews, and Scopus from inception through February 2013 with no language restriction. References of eligible articles and conference proceedings were hand searched and content experts were contacted to identify any additional studies.

\section{Study Selection}

Randomized trials of endovascular therapy (ET) vs. standard care without ET in patients with acute ischemic stroke were included. Two authors independently screened titles and abstracts, and then conducted full text review of those articles that met the inclusion criteria. To be included in the meta-analysis, trials were requited to report data necessary to calculate a risk estimate for all cause mortality, functional outcome, and/or symptomatic intracranial hemorrhage.

This is the author's manuscript of the article published in final edited form as: Hunter, B. R., \& Shardhul, S. V. (2015). Does Endovascular Therapy Benefit Patients With Acute Ischemic Stroke? Annals of Emergency Medicine, 65(3), 288-289. http://doi.org/10.1016/j.annemergmed.2014.09.004 


\section{Data Extraction and Synthesis}

Predesigned abstraction forms were used to extract data from individual studies.

Trial authors were contacted for missing data. Study quality was assessed using the Jadad score as well as the Cochrane Risk of Bias Tool. A random effects model was used to produce pooled relative risk estimates with 95\% confidence intervals. Heterogeneity was reported using the $I^{2}$ statistic and the Cochran Q statistic.

\section{RESULTS}

Pooled relative risk (RR) from 5 randomized trials comparing endovascular therapy to standard care.

\begin{tabular}{|l|l|l|l|}
\hline Outcome (90 d) & $\begin{array}{l}\text { \# Patients } \\
\text { Randomized }\end{array}$ & RR (95\% CI) & $\boldsymbol{I}^{\mathbf{2}}$ \\
\hline Mortality & 1197 & $0.98(0.76-1.25)$ & 0 \\
\hline mRS $\mathbf{1}$ & 1163 & $1.02(0.84-1.23)$ & 1 \\
\hline mRS $\leq \mathbf{2}$ & 1163 & $1.02(0.84-1.24)$ & 21 \\
\hline Symptomatic ICH & 1197 & $0.99(0.62-1.58)$ & 0 \\
\hline
\end{tabular}

Among the 1255 potentially relevant articles identified by the search, only 5 were eligible for meta-analysis. Due to the invasive nature of ET, none of the included trials were blinded to participants (i.e., none used a sham intervention), but 4 of 5 blinded the outcome assessors. There were considerable methodological differences between studies. The time from symptom onset to intervention ranged from less than 3 hours up to 8 hours. One trial protocol required all subjects to receive intravenous tissue plasminogen activator (IV tPA) prior to ET, while the other 4 did not. Four of the 5 trials gave IV tPA to all controls, while one trial allowed "standard 
care" with or without IV tPA. Despite the clinical variation in protocols, there was minimal evidence of statistical heterogeneity among the results (Table).

\section{COMMENTARY}

As stroke centers increasingly adopt endovascular therapy (ET), the benefit of doing so remains unproven. The pooled results of this meta-analysis showed no effect on mortality or functional outcomes with ET compared to standard therapy, but the data based on randomized trials is limited. None of the trials attempted to blind participants, although 4 of the 5 trials blinded outcome assessors. ${ }^{1,2-4}$ It is unclear whether the lack of blinding of participants might unintentionally unmask outcome assessors, or could affect mRS assessments in any way. Given that mRS assessments have modest interobserver agreement,, 56 there is a risk of meaningful bias associated with this lack of blinding. Additionally, two of the 5 trials were stopped early. 1,3

The authors of this systematic review looked at multiple patient-centered outcomes (e.g. mRS) and performed several sensitivity and subgroup analyses, yet were unable to find a statistically significant benefit in any group of patients or any iteration of ET. The authors did note that in the subgroup of patients with severe stroke, defined as NIHSS $\geq 20$, there appeared to be a trend toward better mRS with ET $\left(N=271,3\right.$ trials $\left.{ }^{1-3}\right)$. An adequately powered study would be required to confirm or refute this hypothesis. The authors also hypothesize that time to recanalization may be a critical element for ET success, yet the most aggressive study, ${ }^{1}$ in which the intervention group got IV tPA prior to ET within 3 hours, was stopped early for 
futility. An editorial published alongside this review suggests that more advanced neuroimaging may be the key to identifying patients who will preferentially benefit from ET. ${ }^{7}$ Unfortunately, the only randomized trial to test this hypothesis reported negative results. ${ }^{4}$

Enrollment for future studies will have to focus on which patients are likely to benefit from ET and how to identify them.

\section{Systematic Review Source:}

This is a clinical synopsis, a regular feature of the Annals' Systematic Review Snapshots (SRS) series. The source for this systematic review snapshot is: Singh B, Parkaik AK, Prokop LJ, et al. Endovascular Therapy for Acute Ischemic Stroke: A Systematic Review and Meta-analysis. Mayo Clin Proc. 2013;88:1056-1065.

1. Broderick JP, Palesch YY, Demchuk AM, et al. Endovascular therapy after intravenous t-PA versus t-PA alone for stroke. N Engl J Med. 2013;368:893-903.

2. Ciccone A, Valvassori L, Nichelatti M, et al. Endovascular treatment for acute ischemic stroke. N Engl J Med. 2013;368:904-913.

3. Ciccone A, Valvassori L, Ponzio M, et al. Intra-arterial or intravenous thrombolysis for acute ischemic stroke? the SYNTHESIS pilot trial. $J$ Neurointerv Surg. 2010;2:74-79.

4. Kidwell CS, Jahan R, Gornbein J, et al. A trial of imaging selection and endovascular treatment for ischemic stroke. N Engl J Med. 2013;368:914-923.

5. Fearon P, McArthur KS, Garrity K, et al. Prestroke Modified Rankin Stroke Scale Has Moderate Interobserver Reliability and Validity in an Acute Stroke Setting. Stroke. 2012;43:3184-3188.

6. Quinn TJ, Dawson J, Walters MR, et al. Variability in Modified Rankin Scoring Across a Large Cohort of International Observers. Stroke. 2008;39:2975-2979.

7. Liebeskind DS, Cucchiara B. The Quest to Prove Endovascular Stroke Therapy: Searching for the "Sweet Spot" in Patient Selection. Mayo Clin Proc. 2013;88:1039-1041. 